\section{BENEFITS OF A MATERNAL AND CHILD HEALTH TRANSPORT VOUCHER STUDY. A TRANSPORTER'S PERSPECTIVE IN PALLISA DISTRICT IN EASTERN UGANDA}

Aloysius Mutebi, Elisabeth kiracho Ekirapa. Makerere University School of Public Health, KAMPALA, Uganda

10.1136/bmjopen-2015-forum2015abstracts. 132

Background According to the demographic health survey of 2011 done in Uganda, it indicated that maternal mortality had reduced to 310 (UBOS 2011) from 435 in 2006 (UBOS 2006). This improvement is still much higher than the MDG 5 goal. Transport has been identified as one of the major constraints that contribute to the high MMR.

Objectives In Uganda, only 37 per cent of mothers have a skilled attendant at delivery (rural areas), and only 6 per cent of babies born at home get post natal care. Pallisa district is no exception of the high MMR (310). One of the major delays is transportation of pregnant women and reaching the health facility on time.This study sought to increase attended deliveries using a transport voucher to provide free transport to pregnant women going to deliver in both government and private not for profit health units in the intervention area in Pallisa district and also identified the direct benefits to the transporters.

Methods This study was a quasi-experimental trial in 2 rural health sub districts of Pallisa district. There were both an intervention and control area in this district. The transport voucher was only distributed in the intervention area. There were 8 established motorcycle stages in the intervention area serving 10 health units. This study used qualitative methods for data collection using both FGDs and KI interviews among motorcycle operators. Information was collected by trained Research assistants using question guides and recorders.

Result The number of women delivering in health facilities more than doubled since the inception of the study in June 2010. Out of the active transporters managed to buy their own motorcycles during the period of the project while some mentioned that they had managed to pay up for the motorbikes they had acquired on loan before the project's inception. About 26\% mentioned that they had started some income generating activities (IGAs) out of their savings from transporting pregnant women while the remaining $14 \%$ mentioned having benefited generally without any specific area e.g. improving on their houses, paying school fees, dressing decently and offering better care for their families. Majority (95\%) learnt about maternal issues through sensitisation by study staff and this helped them promote ANC, delivery and PNC in the communities, hence becoming communication agents in their communities of residence.

Conclusion The transport voucher project increased maternal and child health awareness among transporters. Transporters in a way benefited from the savings received out transporting women and were able to start IGAs, buy motorcycles or pay up loans received earlier. Using locally available transport providers has been key to improving access to maternal health services under trained personnel. 